\title{
Preparation and application of a molecularly imprinted polymer for determination of glibenclamide residues
}

\author{
RYM LAHSINI ${ }^{1}$ \\ MOHAMED R. LOUHAICHI ${ }^{2}$ \\ NAFAA ADHOUM ${ }^{1}$ \\ LOTFI MONSER ${ }^{1 *}$ \\ ${ }^{1}$ Laboratoire de Chimie Analytique \\ et d'Electrochimie, Université de Carthage \\ Institut National des Sciences Appliquées \\ et de Technologie, Centre Urbain Nord \\ B.P.N676, 1080 Tunis Cedex, Tunisia \\ ${ }^{2}$ Laboratoire National de Contrôle \\ des Médicaments, 1006 Tunis, Tunisia
}

\begin{abstract}
The performance of a molecularly imprinted polymer (MIP) as selective packing material for solid-phase extraction (SPE) of residual glibenclamide in an industrial process was investigated. MIP was prepared using 4-vinylpyridine as monomer, ethylenedimethacrylate as cross linker, 2,2'-azobis-2-methylpropionitrile as initiator and dimethyl formamide as porogen. Use of acetonitrile as a rebinding solvent allows good recognition of the glibenclamide template. It was found that this polymer can be used for determination of trace levels of glibenclamide with a recovery percentage that could reach $87.1 \%$. Furthermore, the synthesized MIP showed higher selectivity towards glibenclamide than other compounds such as glimepiride and metformine. The synthesized MIP enabled direct determination of the target contaminant after an enrichment step that allowed quantification of glibenclamide at a concentration as low as $0.016 \mathrm{mg} \mathrm{L}^{-1}$. Combination of high performance liquid chromatography with MIP-SPE could be successfully used for quality control of pharmaceuticals during the cleaning process in the production of dry drug forms.
\end{abstract}

Keywords: glibenclamide, molecular imprinted polymer, solid phase extraction, HPLC

Glibenclamide is an antidiabetic drug used for the treatment of diabetes type II. The chemistry of this product makes its analysis difficult especially at low concentrations (1, 2 ). The analytical method used in validation of the cleaning process should fulfil the following requirements: the ability to detect the target substance(s) at levels consistent with the acceptance criteria and the ability to detect the target substance(s) in the presence of other materials that may also be present in the sample (selectivity).

Molecularly imprinted polymer (MIP), as a new specific recognition synthetic polymer (3), could be used as a novel support for solid phase extraction (SPE) of glibenclamide residues. Separation depends on interactions between the template and polymer

\footnotetext{
* Correspondence; e-mail: lotfi.monser@insat.rnu.tn
} 
synthesized from monomer(s), cross linker and porogen. The interactions can involve covalent or non-covalent bonding $(4,5)$. Consequently, these polymeric materials recognize and bind, selectively, only the template molecules. Hence, the choice of the chemical entities used to synthesize MIP plays a crucial role since these compounds have a significant influence on the morphology, physicochemical properties and performance of these polymeric materials $(6,7)$.

In our case, a new MIP was synthesized and used as solid phase support for glibenclamide extraction. Furthermore, its selectivity was evaluated by equilibrium binding and rebinding experiments. Finally, glibenclamide-MIP was applied to demonstrate the level of a pharmaceutical industry cleaning process.

\section{EXPERIMENTAL}

\section{Chemicals}

Glibenclamide, glimepiride and metformine (Fig. 1) were kindly provided by Siphat (Tunisia). Ethylenedimethacrylate (EDMA), 4-vinylpyridine (4-VP) and AIBN [2,2'-azobis-2-methylpropionitrile) were purchased from Sigma Aldrich (France). Acetonitrile, dimethylformamide and methanol were purchased from Scahrlau (France). Ultra pure water corresponding to European Pharmacopea (8) requirements was provided from Siphat (Tunisia). Drug tablets (Glib 5) were also provided from Siphat.

\section{Apparatus and chromatographic conditions}

High performance liquid chromatographic analysis was performed on a Waters Alliance HPLC system, model Waters 2695, consisting of a quaternary pump, a Rheodyne injector and a dual wavelength detector model Waters 2487 (all from the USA). Separation was performed according to European Pharmacopea (8) using a Sunfire C18 column (250 x $4.6 \mathrm{~mm}$ i.d., $5 \mu \mathrm{m}$ particle size). The mobile phase was a mixture of $45 \%$ phase A and $55 \%$ phase $\mathrm{B}$. The mobile phase A consisted of acetonitrile/water $(5: 95, V / V)$ containing $0.2 \%$ triethylamine and adjusted to $\mathrm{pH} 3.0$, while mobile phase B consisted of mobile phase $\mathrm{A}$, water and acetonitrile $(2: 6.5: 91.5, \mathrm{~V} / \mathrm{V} / \mathrm{V})$. The flow rate was $1 \mathrm{~mL} \mathrm{~min} \mathrm{~m}^{-1}$ and the injection volume was $20 \mu \mathrm{L}$.

\section{Preparation of the molecularly imprinted polymer}

A non-covalent molecular imprinting approach was followed to prepare the MIP and the non-imprinted polymer (NIP). The pre-polymerization mixture of glibenclamide (template) (25 mg, $0.0506 \mathrm{mmol})$ dissolved in DMF (3 mL). 4-VP (monomer) (4 mmol, $453 \mu \mathrm{L}$ ) was added to a previously prepared solution and the mixture was stirred for $5 \mathrm{~min}$ at ambient temperature. To this, EDMA was added $(5 \mathrm{mmol}, 970 \mu \mathrm{L})$ and the mixture was stirred for $5 \mathrm{~min}$. Then AIBN (10 mg) was added and the mixture was stirred for further $10 \mathrm{~min}$. Finally, the mixture was sonicated for $10 \mathrm{~min}$ and sparged with nitrogen for $5 \mathrm{~min}$. After $24 \mathrm{~h}$ of thermopolymerisation at $60^{\circ} \mathrm{C}$, the resultant hard polymer was removed. Then, the rigid polymer was ground with a pestle in a mortar and sieved using a $50-\mu \mathrm{m}$ sieve to obtain fine particles $(30-40 \mu \mathrm{m})$ measured by optical microscopy. 


\section{Polymer washing and cartridge preparation}

To remove the template bound within the polymer matrix, the resulting powder was washed for $30 \mathrm{~min}$ with a mixture of methanol and acetic acid $(90: 10, V / V)$. The washing procedure was repeated until no more glibenclamide could be detected in the washing solution. The particles were then washed with methanol and water to remove residual acetic acid, followed by oven drying. In parallel, a non-imprinted control polymer was also synthesized, in the absence of template, following the same procedure as described above. After the washing step, two cartridges (MIP and NIP) were prepared by introducing $100 \mathrm{mg}$ of the powder into a $10 \mathrm{~mL}$ polypropylene syringe secured by frits. Before use, each cartridge was activated by treatment with the same solvent used in the loading step. In the loading step, $2 \mathrm{~mL}$ of glibenclamide solution $\left(50 \mathrm{mg} \mathrm{L}^{-1}\right)$ prepared in a mixture of acetonitrile and water $(6: 4, V / V)$ was passed through the cartridge. The washing step included $1 \mathrm{~mL}$ of acetonitrile. Finally, glibenclamide was eluted with $1 \mathrm{~mL}$ of methanol.

\section{Batch rebinding studies and adsorption isotherm}

For batch rebinding studies, $50 \mathrm{mg}$ of dry MIP and NIP particles were placed in a 100-mL Erlenmeyer flask and incubated for $24 \mathrm{~h}$ with $50 \mathrm{~mL}$ of glibenclamide solution (100 mg L $\mathrm{m}^{-1}$ ). Different solvent mixtures containing acetonitrile, methanol, tetrahydrofuran or a mixture of acetonitrile and water were tested. After the period of incubation, the supernatant was removed, filtered and analyzed by HPLC in order to measure the residual glibenclamide (amount not bound).

For adsorption isotherm studies, $50 \mathrm{mg}$ of polymer was placed in an Erlenmeyer flask containing $50 \mathrm{~mL}$ of glibenclamide $\left(10-300 \mathrm{mg} \mathrm{L}^{-1}\right)$ prepared in acetonitrile/water $(6: 4$, $V / V)$. The solution was shaken for 24 hours at room temperature at a speed of $150 \mathrm{rpm}$. Upon equilibration, all samples were filtered through a $0.45-\mu \mathrm{m}$ filter to minimize the interference of particles during analysis. The residual concentration of glibenclamide was analyzed by HPLC. The amount of glibenclamide was determined from the difference in concentrations at the beginning and at the end of each batch test.

\section{Sampling of glibenclamide and testing of the equipment surface}

Swab sampling technique was used and sites were chosen with care in an attempt to cover the entire equipment surface area. To determine the potential carryover into the subsequent product, it was important that, as a minimum, 16 swab sites from worst case locations on the equipment were selected according to pharmacopeial guidelines (8). Before analysis, $10 \mathrm{~mL}$ of rinse solution was loaded onto the conditioned MIP cartridge. The amount of glibenclamide found in $10 \mathrm{~mL}$ rinsate was selectively retained by the MIP cartridge. After a washing step with $1 \mathrm{~mL}$ acetonitrile, glibenclamide was recovered with $1 \mathrm{~mL}$ methanol.

\section{RESULTS AND DISCUSSION}

\section{Recognition ability of MIP}

In order to evaluate the binding affinity and to establish the optimum conditions under which the template could be recognized by the corresponding MIP, a standard so- 
<smiles>CCC1=C(C)CN(C(=O)NCCc2ccc(S(=O)(=O)NC(=O)NC3CCCCC3)cc2)C1=O</smiles>

Fig. 1. Chemical structure of glibenclamide, glimepiride and metformine.

lution of glibenclamide was initially prepared in various solvents such as dimethylformamide, methanol and acetonitrile. Results (Fig. 2) showed that the prepared polymer allowed better selectivity for glibenclamide ( $\geq 90 \%)$ with acetonitrile as a solvent than with methanol or dimethylformamide. Furthermore, the results obtained illustrate that MIP showed higher affinity for glibenclamide than NIP, indicating that MIP was really imprinted (Fig. 3). This imprinting phenomenon could be explained by the hydrogen bond between glibenclamide proton and pyridine group of the monomer $(9,10)$.

\section{Adsorption isotherms}

Adsorption isotherms are useful in understanding the adsorption interaction mechanism of glibenclamide with the MIP surface. The binding isotherms of glibenclamide (Fig. 4) show that the amount of glibenclamide bound to the MIP at equilibrium increased with concentration until reaching saturation at approximately $45 \pm 1 \mathrm{mg} \mathrm{g}^{-1}$. In con-

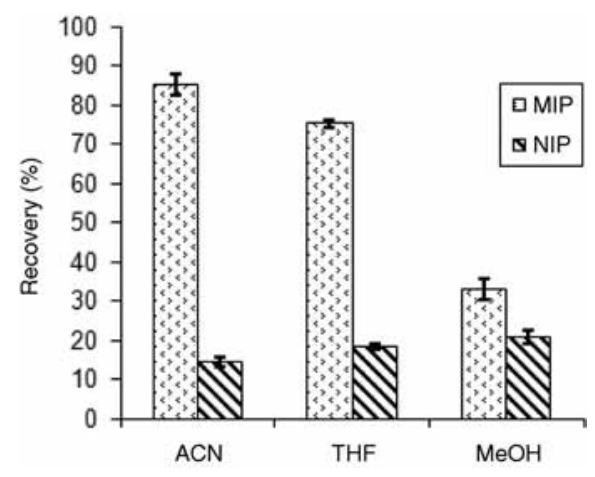

Fig. 2. The effect of acetonitrile, dimethylformamide and methanol as the rebinding solution on glibenclamide rebinding percentage using MIP and NIP (mean \pm SD, $n=3$ ). 


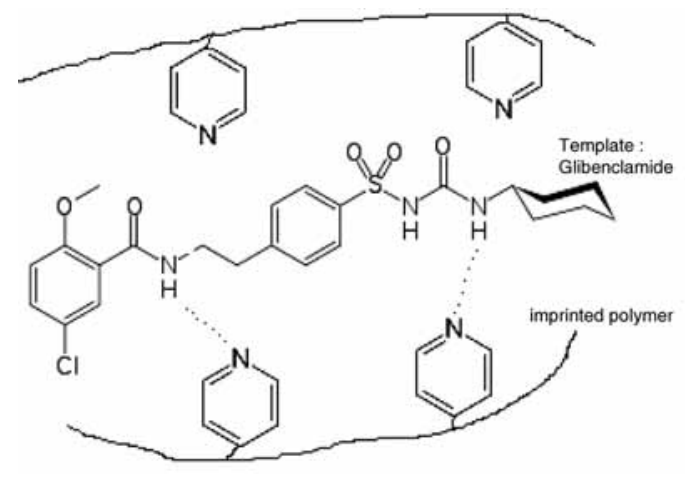

Fig. 3. Illustration of glibenclamide-imprinted polymer and its interactions.

trast, the adsorption amount of glibenclamide with NIP did not exceed $12 \mathrm{mg} \mathrm{g}^{-1}$. These results suggest that the binding to the polymer was mostly caused by specific binding to a limited number of binding sites in the polymer network, rather than by non-specific adsorption (11).

The adsorption isotherm is a function that describes the relationship between the equilibrium concentration of bound $(B)$ and free $(F)$ analyte in the polymer. The re-arranged Freundlich model describes $B$ as a power function of $F$, according to:

$$
B=a F^{m}
$$

or it could be logarithmically transformed into a linear form.

The two parameters, $a$ and $m$, reflect the distribution of binding sites of different binding strengths which are present in the polymer. Term $a$ is a Freundlich parameter related to the binding affinity and term $m$ is the heterogeneity index with values from zero to one, one indicating homogeneity and zero indicating heterogeneity of the sites. This empirical equation is suitable for highly heterogeneous surfaces $(12,13)$.

The obtained data of glibenclamide adsorption equilibrium (Fig. 4) was analyzed using the Langmuir, Schatcard and Freundlich isotherm models. The experimental data into better transformed Freundlich isotherm model than the Langmuir or Schatcard as the experimental data fall on a straight line when plotted in a $\log \mathrm{B}$ vs. $\log \mathrm{F}$ form (Figs. $4 \mathrm{~b}, \mathrm{c})$ allowing for the determination of experimental sorption parameters $\left(R^{2}, m, a\right)$. Data obtained for the Freundlich model concerning MIP and NIP are presented in Table I. The heterogeneity index $m$ obtained for NIP is higher than that of MIP, which is in agreement with previous studies $(14,15)$. As can be seen from the results, the lower $m$ value of MIP indicates a more heterogeneous property than that of NIP. These observations could be attributed to the formation of imprints on the MIP surface. However, the obtained value of $m$ for NIP is sufficiently distant from the unity explaining the role of heterogeneity of the adsorption sites. Within this diversity, we can suppose that certain adsorption sites of NIP have an affinity towards glibenclamide equivalent to that of sites found in MIP. 
a)

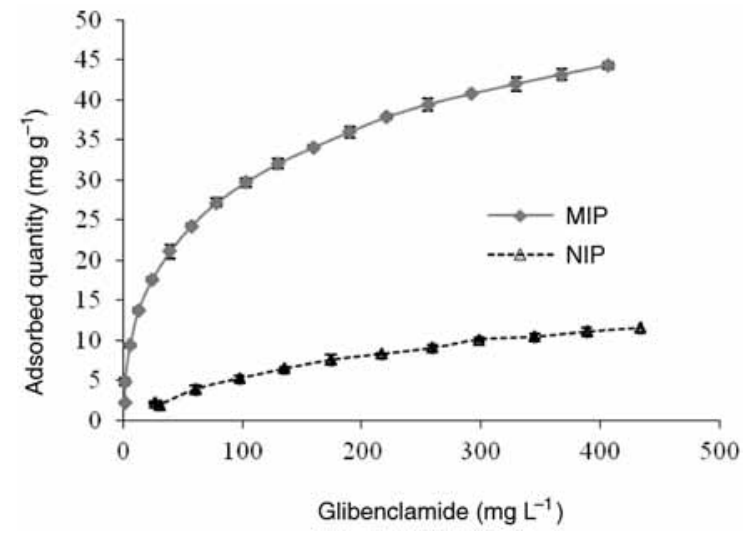

b)

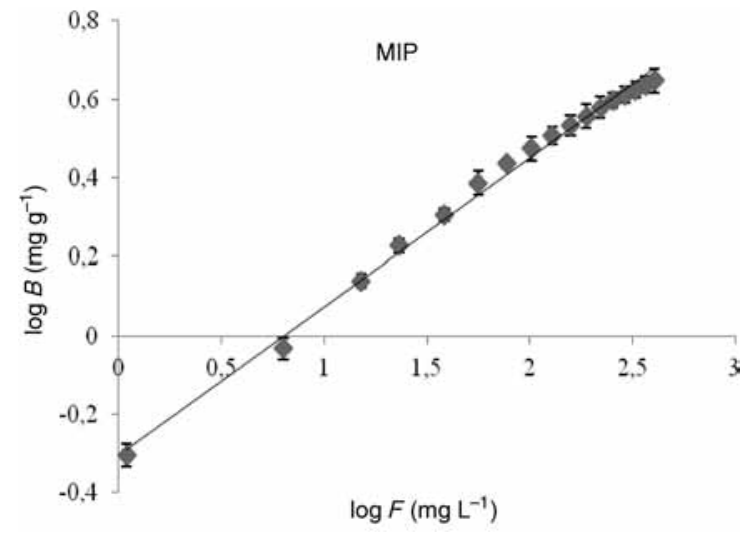

c)

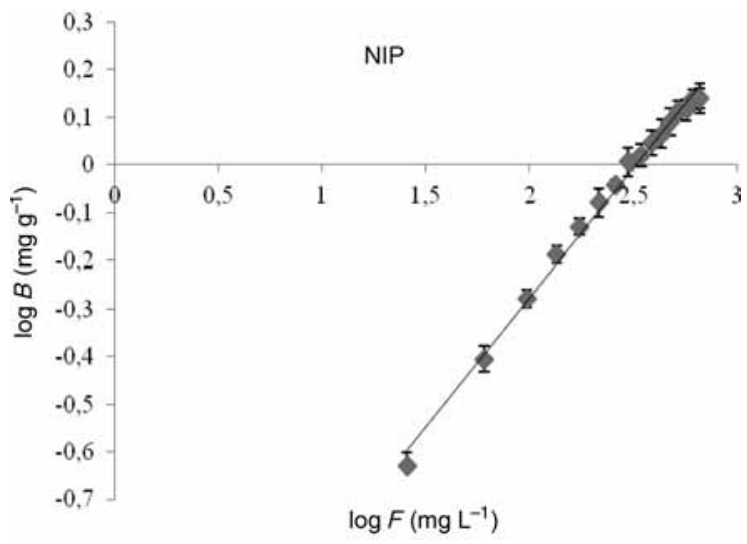

Fig. 4. a) Adsorption isotherm of glibenclamide onto MIP and NIP; b) and c) The re-arranged Freundlich model with MIP and NIP (mean \pm SD, $n=3$ ). 


\section{Application of SPE to HPLC analysis}

The selectivity of MIP for glibenclamide was investigated using glimepiride and metformine as interfering compounds. HPLC results (Fig. 5a) showed the separation of glibenclamide, glimepiride and metformine from a standard mixture before and after MIP-

a)

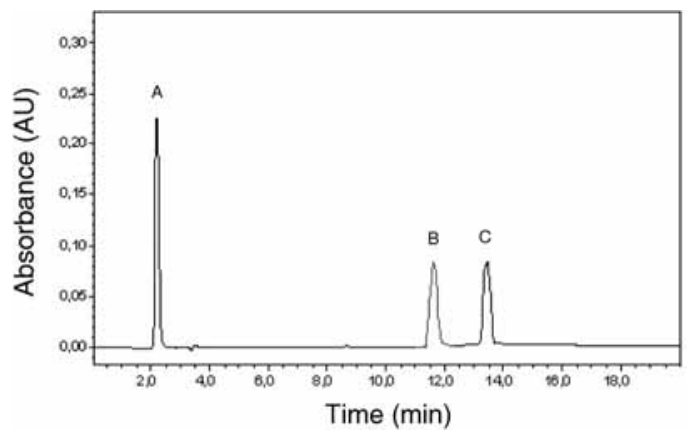

b)

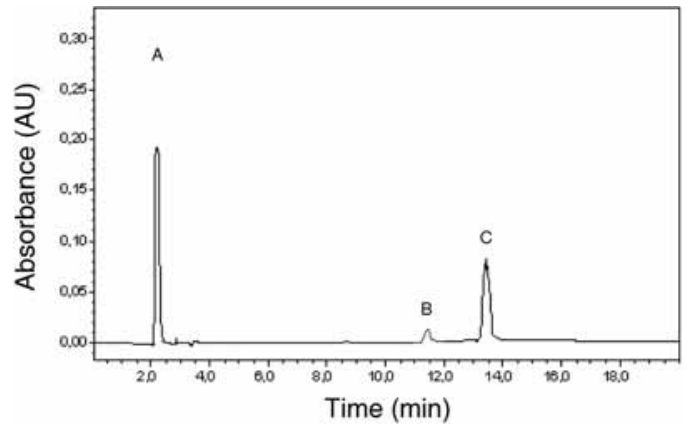

c)

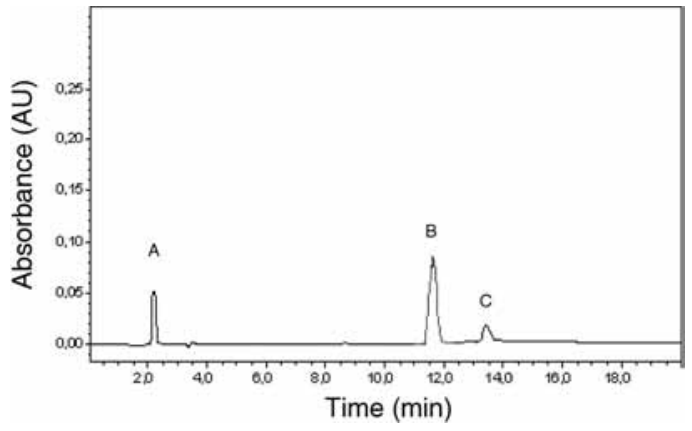

Fig. 5. HPLC chromatograms of standard mixture solutions: a) initial mixture before MIP-SPE, b) mixture after elution through MIP's cartridge and c) mixture after polymer washing with acetonitrile and elution with methanol. Chromatographic conditions as indicated in the experimental part. A - metformine, B - glimepiride, C - glibenclamide. 
-SPE. These results showed the selectivity of the chromatographic method (Table II) as well as the selectivity of the MIP cartridge. This shows that glibenclamide was more strongly retained washed MIP cartridge (Figs. 5b, c) than glimepiride and metformine.

\section{Limit of detection (LOD) and limit of quantification (LOQ)}

The limit of detection and quantitation were calculated as a signal-to-noise ratio $(S / N)$ of 3:1 and 10:1, respectively. The $L O D$ of glibenclamide was found to be $0.04 \mathrm{mg} \mathrm{L}^{-1}$ and the LOQ $0.15 \mathrm{mg} \mathrm{L}^{-1}$.

\section{Recovery study}

The capacity of a MIP corresponds to the amount of a glibenclamide that can be retained on the MIP under given conditions. It was investigated by measuring the recoveries on the MIP for trace concentrations of glibenclamide. It was performed after percolation of $10 \mathrm{~mL}$ of different concentrations of glibenclamide $\left(10-100 \mu \mathrm{g} \mathrm{L}^{-1}\right)$ through a cartridge of MIP. After a washing step with $1 \mathrm{~mL}$ of acetonitrile, glibenclamide was eluted with $1 \mathrm{~mL}$ of methanol. Fig. 6 shows that recovery obtained with glibenclamide-MIP cartridge varied between 63.4 and $87.1 \%$.

\section{Assay after the cleaning process in a production line}

After the production of glibenclamide tablets (Glib 5), a part of the equipment surface $\left(10 \mathrm{~cm}^{2}\right)$ was wiped with acetonitrile and the rinsing solution was analyzed by HPLC. The results obtained (Fig. 7a) showed that no glibenclamide could be detected (concentration

Table I. Freundlich parameters for MIP and NIP

\begin{tabular}{cccc}
\hline Polymer & $R^{2}$ & $m$ & $a$ \\
\hline MIP & 0.993 & 0.367 & 0.285 \\
NIP & 0.976 & 0.582 & 1.466 \\
\hline
\end{tabular}

$R^{2}$ - coefficient of determination; $m$ (slope) and $a$ (intercept) of a line.

Table II. Chromatographic parameters

\begin{tabular}{lccc}
\hline \multicolumn{1}{c}{ Parameter } & Metformine & Glimepiride & Glibenclamide \\
\hline Theoretical plates $(N)$ & 733 & 5880 & 6881 \\
Capacity factor $\left(k^{\prime}\right)$ & 2.36 & 21.82 & 25.75 \\
Selectivity $(\alpha)^{\mathrm{a}}$ & - & 6.794 & 1.172 \\
Resolution $\left(R_{\mathrm{s}}\right)^{\mathrm{a}}$ & - & 23.08 & 3.16 \\
$\operatorname{HETP}(\mu \mathrm{m})^{\mathrm{b}}$ & 341 & 43 & 36 \\
\hline
\end{tabular}

a With respect to previous peak.

b HETP - height equivalent to the theoretical plate. 


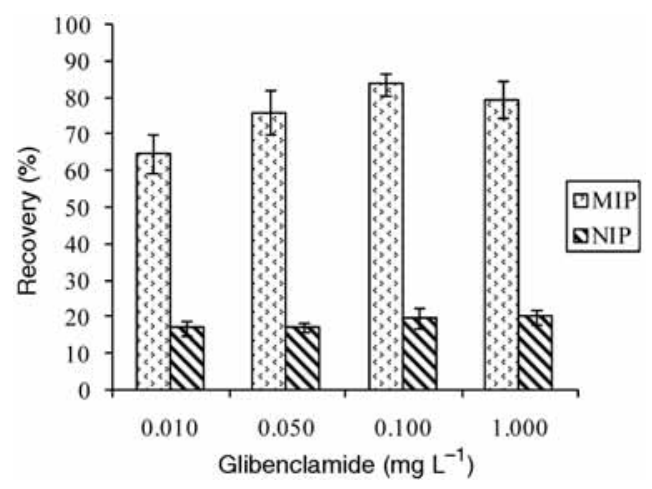

Fig. 6. Recovery of glibenclamide from standard solutions using MIP and NIP (mean $\pm \mathrm{SD}, n=3$ ).

$<L O D$, namely $\left.0.04 \mathrm{mg} \mathrm{L}^{-1}\right)$. Therefore, a step of MIP-enrichment was used. In this step and before HPLC analysis, $10 \mathrm{~mL}$ of rinse solution was loaded onto the conditioned MIP cartridge. After a washing step with $1 \mathrm{~mL}$ acetonitrile, glibenclamide was recovered with $1 \mathrm{~mL}$ methanol. Fig. $7 \mathrm{~b}$ shows a highly detectable and quantifiable glibenclamide peak, indicating that glibenclamide was present in the rinse solution. It was found that this

a)

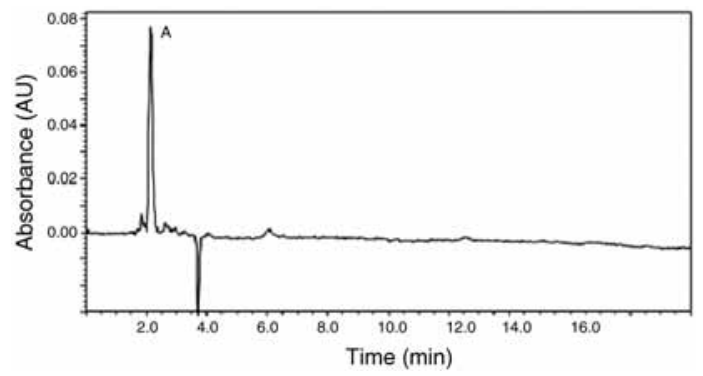

b)

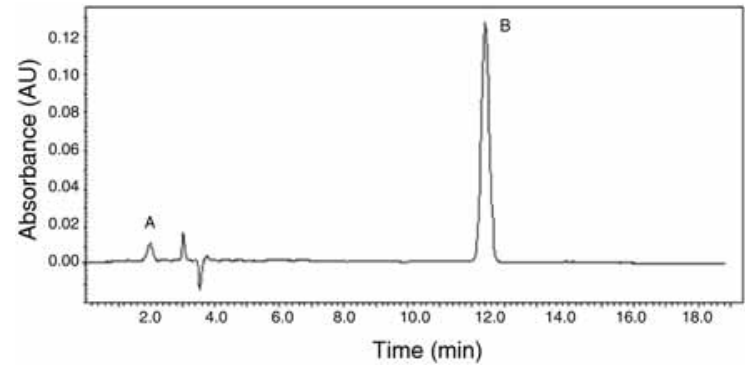

Fig. 7. Chromatograms of rinse solution: a) without MIP-SPE, b): after MIP-SPE enrichment and elution with methanol. Chromatographic conditions as indicated in the experimental part. A - metformine, B - glimepiride. 
concentration of glibenclamide was $0.016 \mathrm{mg} \mathrm{L}^{-1}$. These results indicate that residual glibenclamide was present in the rinse solution, but could not be detected if the analysis of rinse solution was done without a MIP-enrichment step.

\section{CONCLUSIONS}

The applied worst-case approach for pharmaceutical safety and main risks assessment helps figure out the relevant safety issues based on multi-product manufacture cross-contamination. In a preventive action, a new molecularly imprinted polymer was synthesized and used as solid phase support for monitoring glibenclamide residues. The synthesized glibenclamide-MIP showed higher selectivity and enrichment ability towards glibenclamide than glimepiride and metformine. The percentage of rebinding and selectivity were assessed by solid phase extraction and a specific adsorption capacity of $45 \mathrm{mg} \mathrm{g}^{-1}$ of glibenclamide on MIP was estimated. Glibenclamide-MIP SPE cartridge was successfully applied in the evaluation of equipment surface during a pharmaceutical industry cleaning process, prior to HPLC analysis. This could be a preventive action during multi-product manufacture.

\section{REFERENCES}

1. F. Bandarkar and I. Khattab, Simultaneous estimation of glibenclamide, gliclazide, and metformin hydrochloride from bulk and commercial products using a validated ultra fast liquid chromatography technique, J. Liq. Chromatogr. Rel. Techn. 33 (2010) 1814-1830; DOI: 10.1080/10826076. 2010.532704 .

2. P. Chaturvedi and R. Sharma, Development and validation of an RP-HPLC method for simultaneous analysis of a three-component tablet formulation containing metformin hydrochloride, pioglitazone hydrochloride, and glibenclamide, Acta Chromatogr. 20 (2008) 451-461; DOI: 10.1556/ AChrom.20.2008.3.11.

3. G. Vasapollo, R. Del Sole, L. Mergola, M. R. Lazzoi, A. Scardino, S. Scorrano and G. Mele, Molecularly imprinted polymers: Present and future prospective, Int. J. Mol Sci. 12 (2011) 5908-5945; DOI: $10.3390 /$ ijms12095908.

4. S. Piletsky, E. Piletska, K. Karim, G. Foster, C. Legge and A. Turner, Custom synthesis of molecular imprinted polymers for biotechnological application: Preparation of a polymer selective for tylosin, Anal. Chim. Acta 504 (2004) 123-130; DOI: 10.1016/S0003-2670(03)00814-6.

5. Y. Lu, C. Li, X. Liu and W. Huang, Molecular recognition through the exact placement of functional groups on non-covalent molecularly imprinted polymers, J. Chromatogr. A 950 (2002) 89-97; DOI: 10.1016/S0021-9673(02)00058-4.

6. A. Kloskowski, M. Pilarczyk, A. Przyjazny and J. Namiesnik, Progress in development of molecularly imprinted polymers as sorbents for sample preparation, Crit. Rev. Anal. Chem. 39 (2009) 43-58; DOI: 10.1080/10408340802570223.

7. R. Garcia, M. Cabrita and Ana Maria Freitas, Application of molecularly imprinted polymers for the analysis of pesticide residues in food - A highly selective and innovative approach, Am. J. Anal. Chem. 2 (2011) 16-25; DOI: 10.4236/ajac.2011.228119.

8. European Pharmacopoea, 7th ed., Council of Europe, Strasbourg 2008. 
9. V. Pichon, Selective sample treatment using molecularly imprinted polymers, J. Chromatogr. A 1152 (2007) 41-53; DOI: 10.1016/j.chroma.2007.02.109.

10. J. Bastide, J. Cambon, F. Breton, S. Piletsky and R. Rouillon, The use of molecularly imprinted polymers for extraction of sulfonylurea herbicides, Anal. Chim. Acta 542 (2005) 97-103; DOI: 10.1016/j.aca.2005.02.054.

11. M. Castro López, M. Cela Pérez, M. Dopico García, J. López Vilarino, M. González Rodríguez and L. Barral Losada, Preparation, evaluation and characterization of quercetin-molecularly imprinted polymer for preconcentration and clean-up of catechins, Anal. Chim. Acta 721 (2012) 68-78; DOI: $10.1016 /$ j.aca.2012.01.049.

12. G. Rushton, C. Karns and K. Shimizu, A critical examination of the use of the Freundlich isotherm in characterizing molecularly imprinted polymers (MIPs), Anal. Chim. Acta 528 (2005) 107-113; DOI: 10.1016/j.aca.2004.07.048

13. R. Umpleby, S. Baxter, A. Rampey, G. Rushton, Y. Chen and K. Shimizu, Characterization of the heterogeneous binding site affinity distribution in molecularly imprinted polymers, J. Chromatogr. B 804 (2004) 141-149; DOI: 10.1016/ j.jchromb. 2004.01.064

14. A. Lagha, N. Adhoum and L. Monser, A molecularly imprinted polymer for the selective solid-phase extraction of ibuprofen from urine samples, Open Anal. Chem. J. 4 (2011) 7-13; DOI: 10.2174/ 1875038901004010007.

15. R. Umpleby, S. Baxter, M. Bode, J. Berch, R. Shah and K. Shimizu, Application of the Freundlich adsorption isotherm in the characterization of molecularly imprinted polymers, Anal. Chim. Acta 435 (2001) 35-42; DOI: 10.1016/S0003-2670(00)01211-3. 